\title{
Locking plate and fibular allograft augmentation in unstable fractures of proximal humerus
}

\author{
Fabrizio Matassi ${ }^{\mathrm{a}, *}$, Renzo Angeloni ${ }^{\mathrm{b}}$, Christian Carulli ${ }^{\mathrm{a}}$, Roberto Civinini ${ }^{\mathrm{a}}$, Leonardo Di Bella ${ }^{\mathrm{b}}$, \\ Birgit Redl ${ }^{\text {a }}$, Massimo Innocenti ${ }^{\text {a }}$ \\ ${ }^{a}$ First Orthopedic Clinic, Department of Special Surgical Science, University of Florence, CTO-Largo Palagi 1, 50139 Florence, Italy \\ b 3rd General Orthopedic Unit - Azienda Ospedaliera Careggi, CTO - Largo Palagi 1, 50139 Florence, Italy
}

\section{A R T I C L E IN F O}

\section{Article history:}

Accepted 31 July 2012

\section{Keywords:}

Proximal humeral fracture

Fibular allograft

Locking plate

Medial support

Endosteal implant
S U M M A R Y
Introduction: The use of an intramedullary fibular allograft together with a locking plate fixation has been
recently described in biomechanical studies to provide additional medial support and prevent varus
malalignment for displaced proximal humeral fractures with promising results. The aim of this study was
to evaluate the clinical and radiographic outcome of a locking plate with fibular allograft augmentation in
unstable humeral fractures.
Methods: We prospectively assessed the functional outcome and complications in 17 patients with
proximal humeral fractures with disrupted medial column, treated with a locking plate and a fibular strut
graft. The median patient age was 62 years. Postoperative assessments included radiographic imaging,
range of motion, pain according to the visual analogue scale (VAS), Short Form (SF36) Health Survey,
Constant-Murley and Disabilities of the Arm, Shoulder and Hand (DASH) shoulder scores as well as return
to previous occupation and complications.
Results: No patients were lost to follow-up and no major complications were recorded. There was no
collapse of the humeral head more than 2 mm, osteonecrosis or screw penetration of the articular
surface. All fractures healed clinically and radiographically. After an average follow-up of 13 months, the
mean Constant score was 79 points. The mean active flexion was $149^{\circ}$; extension, $47^{\circ}$; internal rotation,
$40^{\circ}$; external rotation, $65^{\circ}$; and abduction, $135^{\circ}$. The median VAS pain level was 1 point. The median
DASH score was 33 points, and the median SF36 was 83 points.
Conclusion: Locking plate with fibular graft augmentation is a safe and promising technique to support
the humeral head and maintain reduction in the proximal humeral fracture with medial comminution. (c) 2012 Elsevier Ltd. All rights reserved.

\section{Introduction}

Displaced proximal humeral fractures remain difficult to treat, mainly because achieving stable fixation that maintains intraoperative reduction is unpredictable. Locking plate fixation has the potential to provide greater fixation in the proximal humerus than standard internal fixation techniques, offering a greater load to failure and the requirement for less soft-tissue dissection along the humeral shaft. ${ }^{1}$ However, some studies show variable results with high rates of screw perforation of the articular surface or varus collapse of the fracture, especially in osteoporotic bone or in fractures with medial metaphyseal comminution. ${ }^{2-8}$

\footnotetext{
* Corresponding author. Tel.: +39055 4376841; fax: +39055 432145

E-mail address: fabriziomatassi@gmail.com (F. Matassi)
}

Adequate mechanical support of the medial column may be obtained by achieving an anatomically stable reduction with a medial cortical contact or, in the case of medial comminution, by placing a superiorly directed oblique locking screw in the inferomedial region of the proximal fragment. Indeed, studies show that fractures treated with either anatomic reduction or screws in the inferomedial humeral head for which no medial column support was obtained had a high incidence of failure. , $^{3-12}$

An intramedullary fibular allograft used together with a locking plate fixation has been recently described in biomechanical studies to provide additional medial support and prevent varus malalignment. $^{13}$

The purpose of this study was to evaluate the clinical and radiographic outcomes of a locking plate with fibular allograft augmentation in unstable humeral fractures. We hypothesised that a fibular strut graft as an endosteal implant would be a safe 
and valuable option to maintain reduction in proximal humeral fractures with metaphyseal comminution.

\section{Materials and methods}

We prospectively followed a selected series of 17 patients (7 men and 10 women) with unilateral displaced proximal humeral fractures treated at our institution between April 2008 and May 2010 by locking plate fixation with intramedullary fibular strut graft augmentation.

Inclusion criteria into the study were closed displaced threeand four-part proximal humeral fractures in adults with disrupted medial hinge and significant metaphyseal comminution or insufficient osseous contact. Open fractures, pathological fractures, unreconstructable head and/or tuberosity fragments and two-part fractures were excluded from the study. The median age of the patients at the time of the operation was 62 years (range, 54-73 years). The mechanisms of injury included a fall while walking (13 patients), motorcycle accident (2), a sports-related accident (1) and a fall from an elevated height (1).

Preoperative evaluation included plain antero-posterior (AP) and lateral X-rays as well as a computed tomography (CT) scan used to evaluate displacement. There were four type A, five type B and eight type $C$ fractures, according to the Arbeitsgemeinschaft für Osteosynthesefragen (AO) classification. ${ }^{14}$ Eleven fractures were 3-part and six fractures were 4-part, according to the Neer classification. ${ }^{15}$

\section{Surgical technique}

All procedures were performed by one of the authors (RA). A complete description of this technique has been published previously. ${ }^{7}$ Patients were placed in the 'beach chair' position on a radiolucent operating table under general anaesthesia. A deltopectoral approach was performed for the first seven cases and an anterolateral deltoid-splitting for the remaining cases. Nonabsorbable sutures (No. 5) were placed in the rotator cuff tendons to allow traction and control of the tuberosity and the humeral head fragment. Two $2.0-\mathrm{mm}$ threaded K-wires were placed in the cancellous surface of the humeral head fragment acting as a 'joystick' for initial reduction. In all cases, the fibular allograft was used as a medial strut augment.

Fibular allograft was cut with a sagittal oscillating saw on the back table to an appropriate length. The fibula was inserted into the canal through the lateral fracture lines and pushed distally beyond the level of the head. It was then medialised maximally to the calcar region to indirectly reduce the medial column and advanced retrograde into the subchondral bone to lift the head superiorly.

The locking plate (for nine cases: Proximal Humerus Internal Locking System PHILOS, Synthes, Mezzovico-Vira, Switzerland; for eight cases: Periarticular Locked Plating System PERI-LOC, Smith \& Nephew, Memphis, TN, USA) was then secured to the lateral humeral shaft distal to the fibula. If the medial column reduction could not be restored and comminution existed, the fibular allograft was used as an indirect reduction tool. A screw pushed the fibula medially until it apposed the medial cortex of the humerus, and indirectly reduced the medial column. If anatomical reduction was achieved, locking screws were placed through the fibula into the humeral head and shaft to secure its position. Meticulous repair of the rotator cuff was carried out, and the wound was closed with a drain placed under negative suction, which was removed after $48 \mathrm{~h}$. Prophylactic intravenous antibiotics were administered until the drain was removed, usually after 24 or 48 h. Postoperatively, the arm was supported in a sling. Pendular movements were started from the first postoperative day and the shoulder was mobilised with passive assisted exercises, followed by active exercises after 3 weeks.

Clinical and radiographic follow-up was performed at 1 month, 2 months, 6 months, 12 months and 2 years, postoperatively. The minimum follow-up was 13 months (average, 28 months; range, 13-56 months).

Clinical examination included range of motion (ROM) of the affected extremity made with a goniometer; and classification of pain, according to the visual analogue scale (VAS), from 0 to 10 ; and the Short Form 36 (SF36) survey. ${ }^{16}$ We also analysed the ConstantMurley shoulder scores for the operated and non-operated shoulders and the Disabilities of Arm, Shoulder and Hand (DASH) shoulder score. ${ }^{17,18}$ Return to previous activities/occupation was also recorded.

Radiologic follow-up included AP and lateral plain X-rays and measurement of the angle between the humeral head and the shaft in the AP view after healing. According to the recommendations of Jiang et al., less than $5^{\circ}$ varus or valgus alignment in the AP view were considered anatomic. ${ }^{4}$ The 'humeral head height' relative to the plate was measured for each radiograph, both initially and at the final follow-up, which allowed for subsequent analysis of loss of reduction. This measurement was done by drawing two lines, both running perpendicular to the shaft of the plate; one was placed at the top edge of the plate and the other was placed at the superior edge of the humeral head, and the distance between these two lines was measured and designated as the head height. ${ }^{3}$ A change in the humeral head height greater than $3 \mathrm{~mm}$ was considered to be a loss of reduction. Complications were recorded, such as collapse of the fracture, screw penetration, avascular necrosis, peri-implant fracture, infection and need for further surgery.

\section{Results}

No patients were lost to follow-up. All fractures healed clinically and radiologically. The mean Constant score at final follow-up was 79 points (range, 56-100 points), representing $83.7 \%$ of the median Constant-Murley score of the unaffected shoulder. The median range of shoulder motion was flexion, $149^{\circ}$ (range, $93-178^{\circ}$ ); extension, $47^{\circ}$ (range, $40-52^{\circ}$ ); internal rotation, $40^{\circ}$ (range, $25-70^{\circ}$ ); external rotation, $65^{\circ}$ (range, $45-90^{\circ}$ ); and abduction, $135^{\circ}$ (range, $80-180^{\circ}$ ). The median VAS pain level was 1 point (range, $0-2$ points). The median DASH score was 33 points (range, $22-52$ points), and the median SF36 was 83 points (range, 67-97 points). Fifteen (88.2\%) patients returned to previous activities or occupations. Two patients experienced restrictions to activities or assumed a different occupation.

Alignment in the AP view was anatomic in 16 patients; however, slight $\left(9^{\circ}\right)$ varus alignment was noted in one patient. There was no collapse of the humeral head or screw penetration of the articular surface for any of the patients. The change in humeral head height between postoperative and the final follow-up X-rays averaged $0.3 \mathrm{~mm}$ (maximum, $1.7 \mathrm{~mm}$ ). Medial cortical continuity was restored in all cases and the fibular allograft showed progressive incorporation into the proximal humerus (Figs. 1-4).

Of the minor complications, one patient had a superficial infection during the early postoperative period. The patient was placed on oral antibiotics for 2 weeks and the infection healed without complication. None of the patients presented with any of the major complications, such as subacromial impingement, neurovascular injury, loosening of implant or osteonecrosis of the humeral head.

\section{Discussion}

The purpose of this study is to analyse the clinical and radiographic outcome of a new technique to treat unstable 


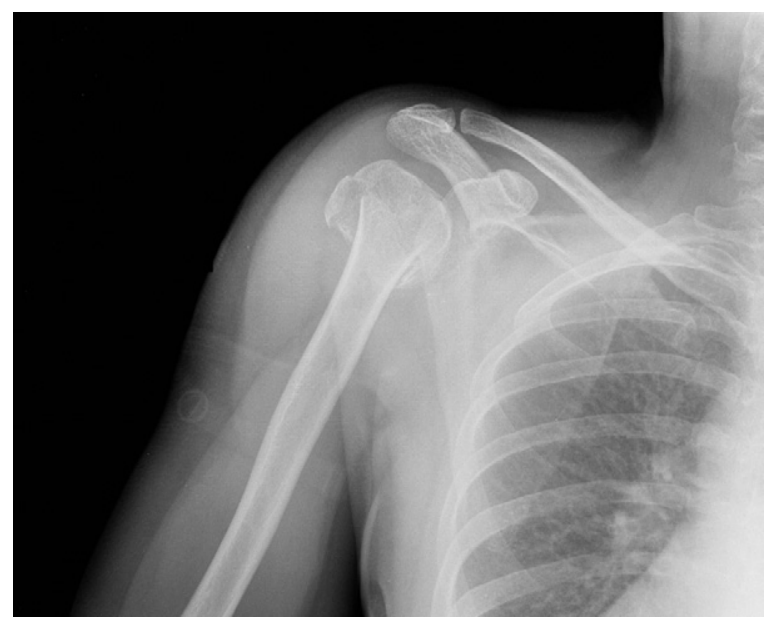

Fig. 1. Anteroposterior X-ray projection of the shoulder in a 68 -year-old female with Neer four-part fracture.

humeral fractures with locking plate and fibular allograft augmentation. We found recovery of good clinical results in all patients, no collapses of humeral head or screw penetration of the articular surface and healing of the fracture with no change in humeral head height between X-rays taken postoperatively and at the final follow-up.

The first clinical experience of this technique to be reported in the literature by Gardner et al. showed encouraging results, with 7 out of 7 fractures healing without a loss of reduction or fixation stability. ${ }^{13}$ Later, Neviaser et al. reported low rates of reduction loss $(2.6 \%)$, screw cut-out $(0 \%)$ and osteonecrosis (2.6\%), as well as high clinical outcome scores in a series of 38 patients with displaced proximal humeral fractures treated with locking plate fixation and an endosteal strut augment. ${ }^{19}$ Biomechanical testing showed that medial support with an intramedullary fibular graft and angular stable fixation increased the overall stiffness of the bone-implant construct and reduced migration of the humeral head fragment, as compared with the locking plate alone. ${ }^{20-23}$

In cadaveric specimens, Chow et al. showed how fibular allograft augmentation could increase the strength of the locking plate to withstand repetitive varus loading. None of the augmented

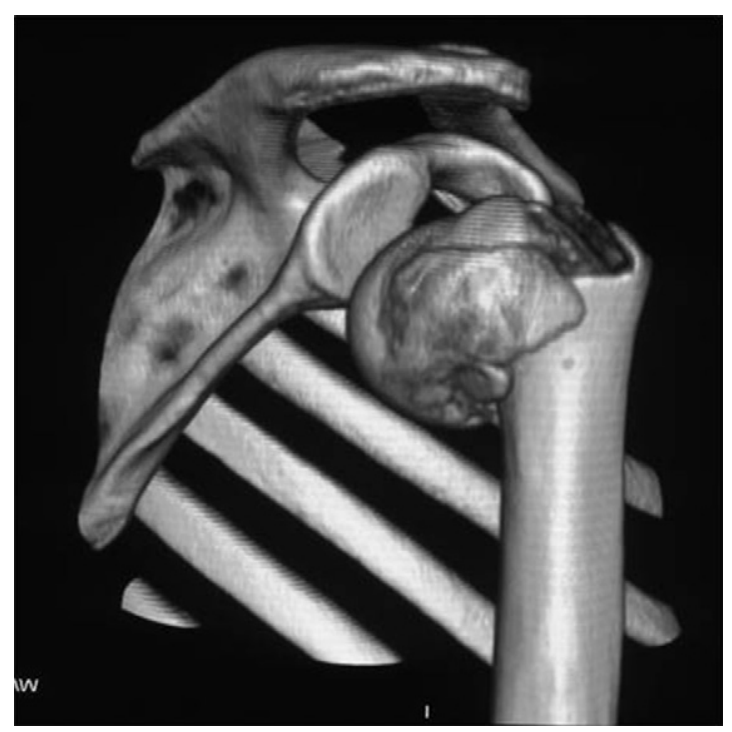

Fig. 2. CT scan shows marked rotation and varus dislocation of humeral head.

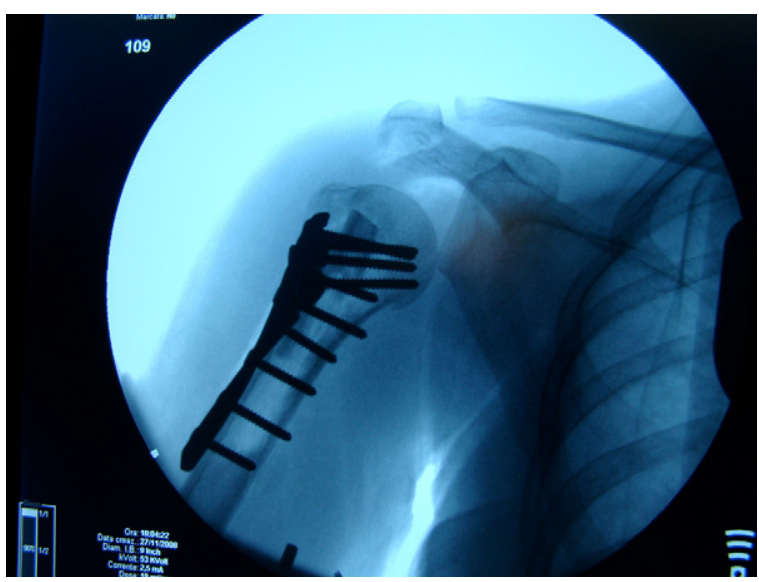

Fig. 3. Intraoperative anteroposterior $\mathrm{X}$ ray following open reduction and internal fixation with locking plate augmented with fibular graft with restoration of humeral head-shaft angle.

constructs failed prior to 25,000 cycles, while 6 of the 8 nonaugmented constructs failed at an average of 6604 cycles. $^{21}$ Furthermore, tests under increasing loads showed that the maximum failure loads and stiffness in the locking plate with intramedullary fibular grafts were significantly higher than those in the locking plate alone. ${ }^{20}$ Osterhoff et al. recently tested a similar construct in synthetic bone of a proximal humerus locking plate augmented with an intramedullary fibular allograft strut. Significantly lower intercyclic fragment motion, lower overall fragment motion and a lower residual gap-distance deformation after 400 cycles of loading was recorded for the group with the fibular graft when compared with the conventional technique. The augmented construct was also stiffer and had a higher ultimate load to failure. $^{23}$

The limitations of our study are as follows. First is the use of two different surgical approaches and two types of locking plate.

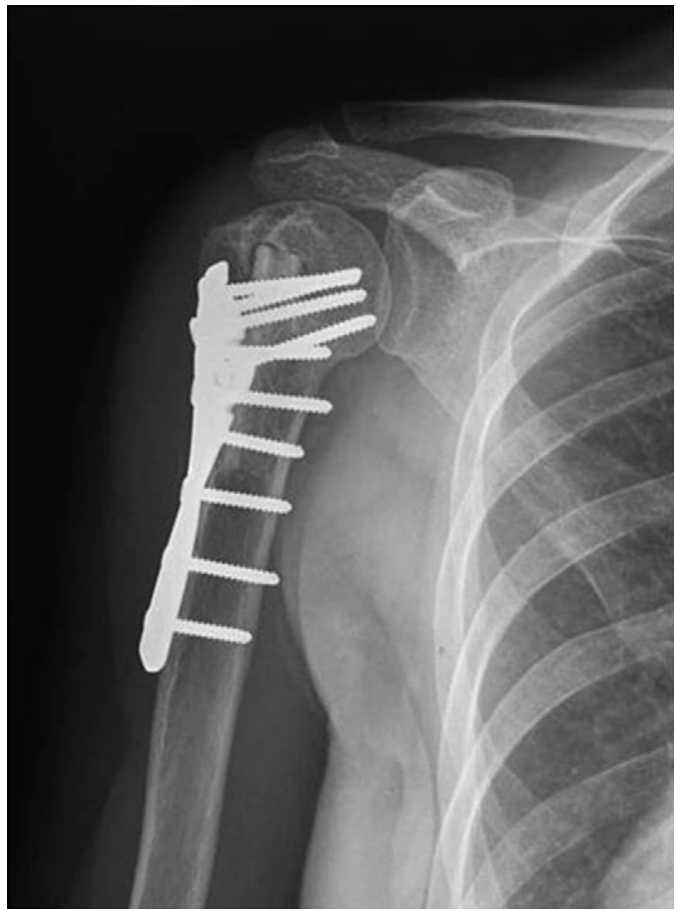

Fig. 4. Anteroposterior radiograph of shoulder at 2 years post-op shows healing of the fracture, no change in humeral head height.

Please cite this article in press as: Matassi F, et al. Locking plate and fibular allograft augmentation in unstable fractures of proximal humerus. Injury (2012), http://dx.doi.org/10.1016/j.injury.2012.08.004 
However, we believe that this could not be a confounding variable for the primary purpose of our study that was to study the mechanical support of a fibular graft in maintaining proximal humeral fracture reduction. Second, we had no control group of similar fractures treated with alternative methods and cannot make direct comparisons of other treatment methods.

Our findings encourage the use of fibular allografts and locking plates for the treatment of proximal humerus fractures with medial comminution to restore the integrity of the medial column, support the humeral head and maintain reduction until fracture healing. Furthermore, the fibula allograft was useful in reduction of the fracture as an indirect tool and at the same time as a mechanical support for the humeral head.

\section{Conclusion}

Locking plate with fibular graft augmentation is a reliable and promising technique to support the humeral head and maintain reduction in the treatment of proximal humeral fractures with medial comminution. We advise the use of this technique in proximal humeral fractures with medial comminution to restore the integrity of the medial column, support the humeral head and maintain reduction until fracture healing. This technique may minimise the most frequent complications reported with proximal humeral locking plates, and allow for early and aggressive physiotherapy.

\section{Conflict of interest}

The authors, their immediate family and any research foundation with which they are affiliated have not received any financial payments or other benefits from any commercial entity related to the subject of this article. The authors declare that there are no outside funding or grants received for this study.

\section{References}

1. Walsh S, Reindl R, Harvey E, Berry G, Beckman L, Steffen T. Biomechanical comparison of a unique locking plate versus a standard plate for internal fixation of proximal humerus fractures in a cadaveric model. Clinical Biomechanics (Bristol Avon) 2006;21:1027-31.

2. Brunner F, Sommer C, Bahrs C, Heuwinkel R, Hafner C, Rillmann P, et al. Open reduction and internal fixation of proximal humerus fractures using a proximal humeral locked plate: a prospective multicenter analysis. Journal of Orthopaedic Trauma 2009;23:163-72.

3. Gardner MJ, Weil Y, Barker JU, Kelly BT, Helfet DL, Lorich DG. The importance of medial support in locked plating of proximal humerus fractures. Journal of Orthopaedic Trauma 2007;21:185-91.
4. Jiang R, Luo CF, Zeng BF, Mei GH. Minimally invasive plating for complex humeral shaft fractures. Archives of Orthopaedic and Trauma Surgery 2007; 127:531-5.

5. Owsley KC, Gorczyca JT. Displacement/screw cutout after open reduction and locked plate fixation of humeral fractures. Journal of Bone and Joint Surgery 2008;90:233-40.

6. Egol KA, Ong CC, Walsh M, Jazrawi LM, Tejwani NC, Zuckerman JD. Early complications in proximal humerus fractures (OTA Types 11) treated with locked plates. Journal of Orthopaedic Trauma 2008;22:159-64.

7. Koukakis A, Apostolou CD, Taneja T, Korres DS, Amini A. Fixation of proximal humerus fractures using the PHILOS plate: early experience. Clinical Orthopaedics and Related Research 2006;442:115-20.

8. Ricchetti ET, Warrender WJ, Abboud JA. Use of locking plates in the treatment of proximal humerus fractures. Journal of Shoulder and Elbow Surgery 2010;19(2 Suppl.):66-75.

9. Krappinger D, Bizzotto N, Riedmann S, Kammerlander C, Hengg C, Kralinger FS. Predicting failure after surgical fixation of proximal humerus fractures. Injury 2011;42(11):1283-8.

10. Lee CW, Shin SS. Prognostic factors for unstable proximal humeral fractures treated with locking-plate fixation. Journal of Shoulder and Elbow Surgery 2009; 18:83-8.

11. Hardeman F, Bollars P, Donnelly M, Bellemans J, Nijs S. Predictive factors for functional outcome and failure in angular stable osteosynthesis of the proximal humerus. Injury 2012;43(2):153-8.

12. Lescheid J, Zdero R, Shah S, Kuzyk PR, Schemitsch EH. The biomechanics of locked plating for repairing proximal humerus fractures with or without medial cortical support. Journal of Trauma 2010;69:1235-42.

13. Gardner M, Boraiah S, Helfet D, Lorich D. Indirect medial reduction and strut support of proximal humerus fractures using an endosteal implant. Journal of Orthopaedic Trauma 2008;22:195-200.

14. Muller ME. The comprehensive classification of fractures of long bones. Berlin, Germany: Springer-Verlag; 1990.

15. Neer 2nd . Displaced proximal humeral fractures. I. Classification and evaluation. Journal of Bone and Joint Surgery 1970;52:1077-89.

16. Bullinger M, Kirchberger I. SF36 questionnaire concerning health status. Gottingen, Bern, Toronto, Seattle: Hogrefe; 1998

17. Constant CR, Murley AH. A clinical method of functional assessment of the shoulder. Clinical Orthopaedics and Related Research 1987;214:160-4.

18. Hudak PL, Amadio PC, Bombardier C, The Upper Extremity Collaborative Group (UECG). Development of an upper extremity outcome measure: the DASH (disabilities of the arm, shoulder and hand). American Journal of Industrial Medicine 1996;29:602-8.

19. Neviaser AS, Hettrich CM, Beamer BS, Dines JS, Lorich DG. Endosteal strut augment reduces complications associated with proximal humeral locking plates. Clinical Orthopaedics and Related Research 2011;469:3300-6.

20. Bae JH, Oh JK, Chon CS, Oh CW, Hwang JH, Yoon YC. The biomechanical performance of locking plate fixation with intramedullary fibular strut graft augmentation in the treatment of unstable fractures of the proximal humerus. Journal of Bone and Joint Surgery - British Volume 2011;98(7):937-41.

21. Chow RM, Begum F, Beaupre LA, Carey JP, Adeeb S, Bouliane MJ. Proximal humeral fracture fixation: locking plate construct \pm intramedullary fibular allograft. Journal of Shoulder and Elbow Surgery 2011;20:1-8.

22. Mathison C, Chaudhary R, Beaupre L, Reynolds M, Adeeb S, Bouliane M Biomechanical analysis of proximal humeral fixation using locking plate fixation with an intramedullary fibular allograft. Clinical Biomechanics 2010;25: 642-6.

23. Osterhoff G, Baumgartner D, Favre P, Wanner GA, Gerber H, Simmen HP, et al Medial support by fibula bone graft in angular stable plate fixation of proximal humeral fractures: an in vitro study with synthetic bone. Journal of Shoulder and Elbow Surgery 2011;20:740-6. 